\title{
Review of: "A scoping review of measurement of violence against women and disability"
}

\author{
April Mackey ${ }^{1}$ \\ 1 University of Saskatchewan
}

Potential competing interests: The author(s) declared that no potential competing interests exist.

This is a scoping review exploring violence against women for those with disability. This is a timely and very important topic and I commend the authors for their work! The authors provide a very insightful and concise discussion of disability as a social construct, as well as a linkage to intersectionality and other conceptual models. The principal findings included implications for a future intersectional approach, as well as highlighted the importance for sex-disaggregated data. These findings are certainly comparable and supported within recent evidence in this area. There were no concerns regarding the scoping review process or rigor of the study and it will be an excellent addition to the knowledge base! There were, however, a few concerns that could improve clarity and readability, which are as follows:

1. The beginning of the background section could be stronger, and the section overall could be more organized. The authors might want to consider a reorganization of the first two paragraphs for clarity, as well as headings within the background section. For example, measurement of women who experience violence who are disabled, and the prevalence of disability among women is discussed throughout but it is difficult to follow each argument as they blend together.

2. Regarding methodology, it was well described, including a concise description of relevant terms for the reader. The authors also provide a comprehensive summary of their search terms, databases etc. A worthy inclusion was suggested reference material from experts in the field. Inclusion and exclusion criteria were thoughtful and clear. Specific detail was paid to sex-specific analyses which is crucial to this topic. Rationales were also provided within the criteria, for example age limit. Study selection and data extraction were clear and thorough. Rationales were provided where appropriate and as the reader, there were no questions left unanswered.

3. The authors may want to include a brief overview of the importance of scoping reviews and the extent of their purview. In addition, the research question should be included at some point early in the articleas this helps the reader to conceptualize your background information, methodology etc. The authors discuss how their research question helped inform their search strategy, but only key words of the question are provided to the reader. The data analysis description was a little difficult to follow as it was mainly just a list of headings the authors chose to focus on in their analysis. The authors may want to consider an alternate way to display this information. 
4. Within the results section, the inclusion of tables 2, 3 and 4 was appreciated - the authors may want to consider adding these as an appendix due to the length and disruption of reading flow.

5. As the section with the most impact for the reader, the discussion would benefit from a reorganization as well as inclusion of headings. There were some very salient points in the discussion that were lost in the reiteration of finding type comments. For example, two major outcomes from this study involved the importance/lack of intersectionality in VAW approaches and the lack of sex-disaggregated data. Readers would benefit from a more wholesome and comprehensive discussion of these in relation to other findings. As it is, these are lost in the lack of headings within the discussion. The authors may want to consider breaking down the discussion section into meaningful chunks - disability defined and discussed in relation to VAW, types of measurement used, intersectionality, sex-disaggregated data. The authors may also want to align this section with the "gaps" provided in the abstract. 